\title{
Detection of child abuse in emergency departments: a multi-centre study
}

\author{
Eveline C F M Louwers, ${ }^{1,2}$ Ida J Korfage, ${ }^{1}$ Marjo J Affourtit, ${ }^{2}$ Dop J H Scheewe, ${ }^{3}$ \\ Marjolijn H van de Merwe, ${ }^{4}$ Francoise A F S R Vooijs-Moulaert, ${ }^{5}$ Claire M C Woltering, ${ }^{6}$ \\ Mieke H T M Jongejan, ${ }^{7}$ Madelon Ruige, ${ }^{8}$ Henriëtte A Moll, ${ }^{2}$ Harry J De Koning ${ }^{1}$
}

\begin{abstract}
1Department of Public Health, Erasmus MC, University Medical Centre Rotterdam, Rotterdam, The Netherlands ${ }^{2}$ Department of Pediatrics, Erasmus MC-Sophia Children's Hospital, Rotterdam, The Netherlands

${ }^{3}$ Department of Pediatrics, IJsselland Hospital, Capelle aan den IJssel, The Netherlands

${ }^{4}$ Advies en Meldpunt Kindermishandeling Zuid-Holland, Gouda, The Netherlands

${ }^{5}$ Department of Pediatrics, Rivas Zorggroep Beatrix Hospital, Gorinchem, The Netherlands

${ }^{6}$ Department of Pediatrics, Reinier de Graaf Gasthuis,

Delft, The Netherlands ${ }^{7}$ Department of Pediatrics, St Franciscus Gasthuis, Rotterdam, The Netherlands ${ }^{8}$ Department of Pediatrics, HagaZiekenhuis Juliana Children's Hospital, The Hague, The Netherlands
\end{abstract}

\section{Correspondence to} Eveline C F M Louwers, Department of Public Health, Erasmus MC, University Medical Centre Rotterdam, Room-Ae 106, PO Box 2040, 3000 CA Rotterdam, The Netherlands; e.louwers@ erasmusmc.nl

Accepted 18 December 2010 Published Online First 30 January 2011

\begin{abstract}
Objective This study examines the detection rates of suspected child abuse in the emergency departments of seven Dutch hospitals complying and not complying with screening guidelines for child abuse.

Design Data on demographics, diagnosis and suspected child abuse were collected for all children aged $\leq 18$ years who visited the emergency departments over a 6 -month period. The completion of a checklist of warning signs of child abuse in at least $10 \%$ of the emergency department visits was considered to be compliance with screening guidelines.
\end{abstract}

Results A total of 24472 visits were analysed, $54 \%$ of which took place in an emergency department complying with screening guidelines. Child abuse was suspected in 52 children (0.2\%). In 40 (77\%) of these 52 cases, a checklist of warning signs had been completed compared with a completion rate of $19 \%$ in the total sample. In hospitals complying with screening guidelines for child abuse, the detection rate was higher $(0.3 \%)$ than in those not complying $(0.1 \%, p<0.001)$. Conclusion During a 6-month period, emergency department staff suspected child abuse in $0.2 \%$ of all children visiting the emergency department of seven Dutch hospitals. The numbers of suspected abuse cases detected were low, but an increase is likely if uniform screening guidelines are widely implemented.

\section{INTRODUCTION}

Child abuse is a serious public health problem with high morbidity and mortality; worldwide, 155000 deaths occur annually in children as a result of abuse or neglect. ${ }^{1}$ Preventing recurrent abuse or recognising early abuse is difficult but essential if long-term effects are to be limited. Although victims of child abuse have higher emergency department use than the general paediatric population, child abuse unfortunately often remains unrecognised in the emergency department. $^{2-6}$

Woodman et al ${ }^{4}$ found consistent evidence that physical abuse affects 1 in 11 children in the UK each year and estimated that about $1 \%$ of all injury-induced child visits to emergency departments are due to physical abuse. In the Netherlands, an estimated 107 200-160 000 children are victims of child abuse annually; however, hospital staff were responsible for only $6 \%$ of the 16156 reports on child welfare in 2008. ${ }^{7-9}$

The overall impression is of suboptimal detection of child abuse in hospitals. Implementing a uniform screening protocol for child abuse in

\section{What is already known on this topic}

- Although child abuse victims have higher emergency department use than the general paediatric population, child abuse unfortunately often remains unrecognised in the emergency department.

- Preventing recurrent abuse or recognising abuse at an early stage is essential to limit its long-term effects.

\section{What this study adds}

- Staff suspected child abuse in only $0.2 \%$ of all children visiting seven Dutch emergency departments over 6 months.

- The detection rate of suspected child abuse was higher in emergency departments complying than not complying with screening guidelines.

- Hospitals should increase compliance with screening guidelines and implement policies to improve emergency department detection of child abuse.

emergency departments could increase detection rates, leading to a decrease in the short- and longterm effects of child abuse. ${ }^{2} 10$ Therefore, the aim of the current study is to assess the detection rates of child abuse in emergency departments in hospitals complying and not complying with uniform screening guidelines and examine the characteristics of cases of suspected abuse.

\section{METHODS}

The province of South-Holland in the Netherlands has a population of 3.5 million people which is served by 22 hospitals. So that the cohort would be representative, data were collected from emergency departments in seven hospitals (a university children's hospital, three teaching hospitals and three rural hospitals) which together have approximately 200000 emergency department visitors annually.

\section{Detection of child abuse}

All children aged $0-18$ years who visited the emergency departments with a new complaint over a 
6-month period were included. We checked all data on cases of suspected abuse, and considered it to be a case of suspected child abuse if emergency department staff noted their suspicions in the medical record. Children who were specifically referred to the hospital with a suspicion of child abuse were not included in the number of cases detected in the emergency department. The definition used for child abuse was any form of threatening or violent physical, mental or sexual interaction with a minor which is perpetrated actively or passively by parents or other persons on whom the minor is dependent and causes or will probably cause physical or mental injury and serious harm to the minor. ${ }^{11}$

All hospitals had a checklist of warning signs of child abuse available in the emergency department. These checklists were either used for all children visiting the emergency department, or, in one hospital, only for children with trauma, and were mostly completed by emergency department nurses. If one of the warning signs on the checklist was ticked, the emergency department nurse informed the treating specialist that the suspicion of child abuse was increased. The frequency of checklist use varied greatly. Compliance with screening guidelines for child abuse in the emergency department was considered to be use of the checklist in at least $10 \%$ of all emergency department visits by children. The numbers of suspected abuse cases detected in the emergency department were compared between hospitals complying and not complying with the screening guidelines.

This study was approved by the Medical Ethical Committee of the Erasmus MC, University Medical Centre Rotterdam.

\section{Data collection and processing}

Data were collected over 6 months in each hospital. In five of the seven hospitals this period covered August 2007 to January 2008. For logistical reasons, the collection period was November 2007 to April 2008 in one hospital and March 2008 to August 2008 in another. For all children data were collected on demographics, reason for the emergency department visit, the referrer, the treating specialist and the diagnosis at discharge. We used emergency department triage systems and (electronic) patient files, and if available, data from the screening checklist for child abuse. Based on all this information, we assessed whether or not emergency department staff suspected child abuse.

\section{Statistical analysis}

The $\chi^{2}$ test was used to compare baseline categorical variables between hospitals complying and not complying with screening policy, between cases screened and not screened for child abuse, and between children with and without suspected abuse. The Wilcoxon rank test was used for the continuous variable age. Analyses were performed using the statistical package SPSS 15.0. Statistical significance was defined as $\mathrm{p}<0.05$.

\section{RESULTS}

\section{Emergency department visitors}

During the 6-month study period, 24472 children aged $\leq 18$ years visited one of the seven emergency departments with a new complaint. Of these, 56\% (13 754) were male, and more than $40 \%$ (10 546) were 4 years of age or younger. The majority $(52 \%)$ visited the emergency department without being referred, and most (60\%) had a surgical problem (eg, a fracture).

Of all children, 54\% visited an emergency department which complied with screening guidelines. The patients in these hospitals were older, less often self-referred, and more often treated by a paediatrician than those who visited hospitals not complying with screening guidelines (table 1).

\section{Screening}

Overall, the checklist was completed for $36 \%$ of emergency department visitors (ranging from 16\% to 56\%) in hospitals complying versus $0.4 \%$ in hospitals not complying with the guidelines. The rate of suspected child abuse was higher in hospitals complying versus hospitals not complying with screening guidelines $(0.3 \%$ vs $0.1 \%, \mathrm{p}<0.001)$ (table 1$)$.

The 4769 children who were screened for child abuse were significantly younger than those not screened $(p<0.001)$. Children screened for abuse were less often self-referrals and

Table 1 Characteristics of all emergency department visitors aged $\leq 18$ years over 6 months in seven Dutch hospitals classified as complying or not complying with screening policy

\begin{tabular}{|c|c|c|c|c|}
\hline & $\begin{array}{l}\text { Hospitals complying with } \\
\text { screening policy }\end{array}$ & $\begin{array}{l}\text { Hospitals not complying with } \\
\text { screening policy }\end{array}$ & Total & p Value ${ }^{*}$ \\
\hline Emergency department visitors & 13109 & 11363 & 24472 & \\
\hline Age in years (range) & $7.6(0-18)$ & $6.0(0-18)$ & $6.9(0-18)$ & $<0.001$ \\
\hline $5-8$ & $2233(17 \%)$ & $1938(17 \%)$ & $4171(17 \%)$ & \\
\hline $9-12$ & $2210(17 \%)$ & $1366(12 \%)$ & $3576(15 \%)$ & \\
\hline $13-18$ & $3829(29 \%)$ & $2157(19 \%)$ & $5986(25 \%)$ & \\
\hline \multicolumn{5}{|l|}{ Referrer } \\
\hline Self-referral & $5505(43 \%)$ & $6937(63 \%)$ & $12442(52 \%)$ & $<0.001$ \\
\hline General practitioner & $2921(23 \%)$ & $2622(24 \%)$ & $5543(23 \%)$ & \\
\hline Other & $4469(35 \%)$ & $1383(13 \%)$ & $5852(25 \%)$ & \\
\hline \multicolumn{5}{|l|}{ Treating specialist } \\
\hline Surgeon & $7616(58 \%)$ & $6924(61 \%)$ & $14540(60 \%)$ & $<0.001$ \\
\hline
\end{tabular}

*Continuous variable (age) calculated with Wilcoxon rank test; categorical variables calculated with the $\chi^{2}$ test. 
Table 2 Characteristics of all emergency department visitors aged $\leq 18$ years over 6 months in seven Dutch hospitals classified by cases screened or not screened

\begin{tabular}{|c|c|c|c|}
\hline & Cases screened for child abuse & Cases not screened for child abuse & p Value* \\
\hline Emergency department visitors & 4769 & 19167 & \\
\hline Age in years (range) & $6.2(0-18)$ & $6.9(0-18)$ & $<0.001$ \\
\hline $0-4$ & 2052 (43\%) & 8352 (44\%) & \\
\hline $5-8$ & $976(21 \%)$ & $3112(16 \%)$ & \\
\hline $9-12$ & $901(19 \%)$ & $2593(14 \%)$ & \\
\hline $13-18$ & $838(18 \%)$ & $4937(26 \%)$ & \\
\hline Sex (male) & $2690(56 \%)$ & 10772 (56\%) & 0.85 \\
\hline \multicolumn{4}{|l|}{ Referrer } \\
\hline Self-referral & $2105(45 \%)$ & 10258 (54\%) & $<0.001$ \\
\hline General practitioner & $1390(30 \%)$ & $4044(21 \%)$ & \\
\hline Other & $1274(27 \%)$ & $4865(25 \%)$ & \\
\hline \multicolumn{4}{|l|}{ Treating specialist } \\
\hline Surgeon & $2800(59 \%)$ & $11432(60 \%)$ & $<0.001$ \\
\hline Paediatrician & $1739(37 \%)$ & $5894(31 \%)$ & \\
\hline Other & $227(5 \%)$ & $1753(9 \%)$ & \\
\hline Suspected child abuse cases in he emergency department & $40(0.8 \%)$ & $12(0.1 \%)$ & $<0.001$ \\
\hline
\end{tabular}

${ }^{*}$ Continuous variable (age) calculated with Wilcoxon rank test; categorical variables calculated with the $\chi^{2}$ test.

Table 3 Diagnoses of suspected cases of child abuse in seven Dutch hospitals over 6 months

\begin{tabular}{llc}
\hline Type of abuse & Diagnosis & Children (n) \\
\hline Physical abuse & & $37(71 \%)$ \\
& Fractures/luxation & 18 \\
& Bruises/wounds/burns/contusion & 15 \\
& Inflicted traumatic brain/head injury & 4 \\
Neglect & & $13(25 \%)$ \\
& Various diagnoses (intoxication, wounds, \\
Sexual abuse & commotiocordis, infection) & $2(4 \%)$ \\
& & \\
\hline
\end{tabular}

were more often treated by a paediatrician. Significantly more cases of suspected abuse arose in children screened than not screened for child abuse (0.8\% vs $0.1 \%, \mathrm{p}<0.001)$ (table 2$)$.

\section{Cases of suspected child abuse}

After excluding 23 children specifically referred with suspected abuse, a suspicion of child abuse arose in $0.2 \%$ (52) of all remaining emergency department visitors. Cases of suspected child abuse were on average 3.9 years old, and $33 / 52$ were aged $\leq 4$ years. In 40 of the 52 cases, a checklist of warning signs of abuse was completed. Most suspicions concerned physical abuse, with fractures being the most often reported diagnosis (table 3 ).

\section{DISCUSSION}

During a 6-month study period, a suspicion of child abuse arose in $0.2 \%$ of children aged $\leq 18$ years who visited the emergency departments of seven hospitals, and in $0.3 \%$ of children aged $\leq 4$ years. The significantly higher detection rate in hospitals complying with screening guidelines for child abuse $(0.3 \%)$ compared to those not complying $(0.1 \%)$ shows the importance of increased situational awareness for improving detection of child abuse. Checklists were completed in 40 (77\%) of the 52 cases of suspected abuse but in only $19 \%$ of the total population, although the use of checklists in suspected cases might have been intentional selection.

Implementation of a structured screening protocol, therefore increasing the situational awareness of child abuse, might result in a higher detection rate of suspected child abuse. A checklist of warning signs of child abuse could be part of such a protocol, but a validated checklist is currently not available. ${ }^{12}$ If a checklist were used, it might result in more cases of suspected child abuse being identified and would be a first step in improving the detection rate of actual cases of child abuse. The use of a checklist in every child visiting the emergency department would result in universal screening to identify a high risk group. ${ }^{13}$ Unfortunately, an increase in the sensitivity of child abuse detection would lead to a decrease in specificity. Therefore, a protocol with clear guidelines on how to manage suspicions of child abuse is required. Where a case is suspected, it is very important that the physician informs the parents about his or her concerns without accusing anyone. Unfortunately, fear among physicians and other emergency department staff of making a false accusation can lead to failure to report cases of suspected abuse.

Screening for child abuse in the emergency department is not standard policy in most countries (eg, USA, Canada and the UK), but did become mandatory in the Netherlands in $2009 .{ }^{14}$ Earlier studies on screening (each including 2000-4422 patients) reported higher detection rates of suspected abuse (range $1.1-1.4 \%$ ) than the $0.3 \%$ rate identified in the present study. ${ }^{15-17}$

We found that the detection rate of suspected child abuse was much higher when a checklist of warning signs of abuse was completed. Comparison of screened cases with those not screened showed that emergency department staff completed the checklist more often in children who were younger, were referred by a general practitioner or were treated for a paediatric complaint.

In the present study, children suspected of being abused were younger than the average child in the emergency department. The younger the child, the more vulnerable he or she is, the higher the risk that an injury requires medical attention, and the higher the chance that emergency department staff suspect abuse. ${ }^{18}$ However, because child abuse can affect children of all ages, emergency department staff must be aware of the risk in all children visiting the emergency department to avoid missing cases of child abuse. ${ }^{19}$

Physical abuse is the most common type of child abuse detected in the emergency department, ${ }^{6}$ as shown in the present 
study. Neglect and emotional and sexual abuse are more difficult to identify in an emergency department setting but also require attention. Overall, child abuse remains an under-reported problem. This can be attributed to, for example, inadequate knowledge and training of professionals regarding recognition of abuse injuries, unwillingness to report suspicions of abuse, and variations in what is considered to be abuse..$^{18}$

Some limitations of the present study need to be addressed. First, we present cases of suspected abuse. Since abuse was not yet confirmed, this could have led to an overestimation of the detection rate of child abuse. Second, cases of suspected abuse might have been missed because only one of the hospitals systematically registered such cases. Finally, for optimal data comparison the same time period should have been used in all hospitals. However, due to logistical problems this was not possible in two of the participating centres.

The strengths of this study are the relatively long observational period, the large number of children, the inclusion of all patients ( $\leq 18$ years old) who visited the emergency departments with a new complaint, and the fact that of the results are representative of various emergency department settings.

In summary, the detection rates of suspected child abuse in children who visited an emergency department were very low $(0.2 \%)$. However, the detection rate of suspected abuse was higher in hospitals where emergency department staff complied with screening guidelines than in hospitals with non-compliant emergency department staff. We recommend that hospitals encourage compliance with screening guidelines, implement strict policies to improve the detection rate of suspected child abuse in emergency departments, and use the results of these interventions to develop an optimal screening protocol for emergency departments. Further research is recommended on how to identify genuine cases of child abuse among the high risk group of suspected cases identified by screening.

Acknowledgements The authors thank the following hospitals for their friendly cooperation: Erasmus MC-Sophia Children's Hospital, IJsselland Hospital, Albert Schweitzer Hospital, Rivas Zorggroep Beatrix Hospital, Reinier de Graaf Gasthuis, St Franciscus Gasthuis and HagaZiekenhuis Juliana Children's Hospital.

Funding This study was funded by the Netherlands Institute for Health Research and Development (ZonMw 633000 23). The authors were completely independent of funders in conducting this study and writing this manuscript.

\section{Competing interests None.}

Ethics approval This study was conducted with the approval of the Medical Ethical Committee of Erasmus MC, Rotterdam, The Netherlands.

Provenance and peer review Not commissioned; externally peer reviewed.

\section{REFERENCES}

1. Gilbert R, Widom CS, Browne K, et al. Burden and consequences of child maltreatment in high-income countries. Lancet 2009;373:68-81.

2. Guenther $\mathbf{E}$, Knight $\mathrm{S}$, Olson LM, et al. Prediction of child abuse risk from emergency department use. J Pediatr 2009;154:272-7.

3. Spivey MI, Schnitzer PG, Kruse RL, et al. Association of injury visits in children and child maltreatment reports. J Emerg Med 2009;36:207-14.

4. Woodman J, Pitt M, Wentz R, et al. Performance of screening tests for child physical abuse in accident and emergency departments. Health Technol Assess 2008;12:1-95.

5. Tenney-Soeiro R, Wilson C. An update on child abuse and neglect. Curr Opin Pediatr 2004;16:233-7.

6. Gilbert R, Kemp A, Thoburn J, et al. Recognising and responding to child maltreatment. Lancet 2009;373:167-80.

7. Euser EM, van ljzendoorn MH, Prinzie P, et al. Prevalence of child maltreatment in The Netherlands. Child Maltreat 2010;15:5-17.

8. Lamers-Winkelman F, Slot NW, Bijl B, et al. Scholieren over mishandeling; Resultaten van een landelijk onderzoek naar de omvang van kindermishandeling onder leerlingen van het voortgezet onderwijs. [School Students About Abuse; Results of a National Survey of the Extent of Child Abuse Among Pupils of Secondary School.] Duivendrecht: PI Research, Vrije Universiteit Amsterdam, Faculteit der Psychologie en Pedagogiek, WODC, 2007.

9. MOgroep Jeugdzorg. Adviezen en meldingen over kindermishandeling in 2008. [Advice and Reports of Child Abuse in 2008.] Utrecht: MOgroep Jeugdzorg, 2009.

10. Ethier LS, Lemelin JP, Lacharité C. A longitudinal study of the effects of chronic maltreatment on children's behavioral and emotional problems. Child Abuse Negl 2004;28:1265-78.

11. Wet op de Jeugdzorg. [Law on Youth Care.] http://wetten.overheid.nl/ BWBR0016637/geldigheidsdatum 19-04-2010\#Hoofdstukl Artikel1 Olaccessed 19 April 2010).

12. Louwers EC, Affourtit MJ, Moll HA, et al. Screening for child abuse at emergency departments: a systematic review. Arch Dis Child 2010;95:214-18.

13. Marteau TM, Kinmonth AL. Screening for cardiovascular risk: public health imperative or matter for individual informed choice? BMJ 2002;325:78-80.

14. Wal van der G. Afdeling spoedeisende hulp van ziekenhuizen signaleert kindermishandeling nog onvoldoende: gebroken arm nog te vaak een ongelukje. [Observation of Child Abuse Still Insufficient at Emergency Departments of Hospitals: Broken Arm Is Too Often Just an Accident.] The Hague: Health Care Inspectorate, 2008

15. Benger JR, Pearce V. Simple intervention to improve detection of child abuse in emergency departments. BMJ 2002;324:780.

16. Pless IB, Sibald AD, Smith MA, et al. A reappraisal of the frequency of child abuse seen in pediatric emergency rooms. Child Abuse Neg/ 1987;11:193-200.

17. Sidebotham PD, Pearce AV. Audit of child protection procedures in accident and emergency department to identify children at risk of abuse. BMJ 1997;315:855-6.

18. Kellogg ND. American Academy of Pediatrics Committee on Child Abuse and Neglect. Evaluation of suspected child physical abuse. Pediatrics 2007;119:1232-41.

19. Trocmé N, MacMillan H, Fallon B, et al. Nature and severity of physical harm caused by child abuse and neglect: results from the Canadian Incidence Study. CMAJ 2003;169:911-15. 